\title{
A tool for examining the role of the zinc finger myelin transcription factor 1 (Myt1) in neural development: Myt1 knock-in mice
}

\author{
Lynn D. Hudson • Elena Romm • Jo Ann Berndt • \\ Joseph A. Nielsen
}

Received: 28 March 2010/ Accepted: 23 November 2010/Published online: 26 January 2011

(C) The Author(s) 2011. This article is published with open access at Springerlink.com

\begin{abstract}
The Myt1 family of transcription factors is unique among the many classes of zinc finger proteins in how the zinc-stabilized fingers contact the DNA helix. To examine the function of Myt1 in the developing nervous system, we generated mice in which Myt1 expression was replaced by an enhanced Green Fluorescent Protein fused to a Codonimproved Cre recombinase as a protein reporter. Myt 1 knock-in mice die at birth, apparently due to improper innervation of their lungs. Elimination of Myt1 did not significantly affect the number or distribution of neural precursor cells that normally express Myt1 in the embryonic spinal cord. Nor was the general pattern of differentiated neurons altered in the embryonic spinal cord. The Mytl knock-in mice should provide an important tool for identifying the in vivo targets of Myt1 action and unraveling the role of this structurally distinct zinc finger protein in neural development.
\end{abstract}

L. D. Hudson $(\bowtie) \cdot$ E. Romm · J. A. Berndt .

J. A. Nielsen

Section of Developmental Genetics, National Institute of Neurologic Disorders and Stroke, National Institutes of Health, Building 1, Room 228, Bethesda,

MD 20892-0166, USA

e-mail: hudsonl1@od.nih.gov

Present Address:

J. A. Nielsen

Center for Devices and Radiological Health, Food

and Drug Administration, Silver Spring, MD, USA
Keywords Oligodendrocytes - Transcriptional networks · Corepressors · Myelinogenesis .

Zinc finger transcription factors

\section{Introduction}

During development, oligodendrocyte precursor cells arise in multiple locations and differentiate into the myelinating cells of the central nervous system. Much progress has been made in the identification of individual transcription factors required for orchestrating the multiple waves of oligodendrocyte development. (Kessaris et al. 2006; Nicolay et al. 2007; Wegner 2008). The myelin transcription factor 1 (Mytl) family represents zinc finger proteins of a structurally novel CCHC class that were originally cloned by their binding to the most abundantly expressed myelin gene in oligodendrocytes: proteolipid protein (Plpl; Kim and Hudson 1992). Myt1 zinc fingers recognize DNA in a distinct manner, with a whole zinc finger sitting snugly in the major groove of double-stranded DNA (Gamsjaeger et al. 2008). This manner of interaction contrasts with the classical GATA-type and steroid hormone zinc finger proteins, which all utilize an $\alpha$-helix to position DNAcontacting residues in the major groove. Myt1 physically associates with the Sin3B protein, a corepressor that modifies chromatin structure by 
binding class I histone deacetylases (HDACs; Romm et al. 2005). Of note, oligodendrocytes contain multiple forms of $\operatorname{Sin} 3 \mathrm{~B}$, both a long form that includes a HDAC binding site and short forms that lack a HDAC binding site. Myt1 binding to target genes can recruit HDAC via Sin3B, with an accompanying localized hypo-acetylation of core histones and resultant transcriptional repression. But in the face of sufficient amounts of Sin3B short forms, the Sin3B-Myt1 complexes bound to target genes may preclude HDAC binding, leading to a state of transcriptional activation. Within the nucleus, Myt1 is located in domains that are distinct from the nucleolus, coiled bodies, and other nuclear subdomains (Armstrong et al. 1995). The discrete punctate pattern of Myt 1 immunostaining coincided with the target PLP gene, which was associated with the nuclear periphery, in half of the cells observed in both progenitors and differentiated oligodendrocytes (Nielsen et al. 2002). Mature oligodendrocytes do not express appreciable amounts of Myt1 and the subcellular distribution of Myt1 shifts shortly before cells stop expressing Myt1; consequently the Myt1 protein in mature oligodendrocytes is transiently detected in the cytoplasmic compartment (Armstrong et al. 1995).

Myt1 modulates the proliferation and differentiation of oligodendrocyte lineage cells in rodents (Nielsen et al. 2004), as observed following the introduction of a dominant negative construct containing the four zinc-finger binding domain of Myt1 into oligodendrocyte primary cultures. Expression of the dominant negative construct inhibited the proliferation of progenitors as well as their differentiation into oligodendrocytes, as assessed by bromodeoxyuridine incorporation, morphology, migratory characteristics, and myelin gene expression. Myt1 alters neuronal differentiation in Xenopus (Bellefroid et al. 1996), and together with the basic-helix-loop-helix protein Neurogenin 3 forms a "feed-forward" expression loop to promote endocrine islet cell differentiation (Ahnfelt-Ronne et al. 2007; Wang et al. 2008). Selective knock-out of Myt1 in the pancreas compromises islet cell differentiation; of note is the induction of the paralogs Myt11 and Myt3 in these animals (Wang et al. 2007). In the nervous system, Myt1 is expressed in neuroepithelial germinal zones in rodent embryos (Kim et al. 1997) and continues to be expressed in germinal zones of adults (Armstrong et al. 1995). Myt1 cannot be detected in cells immunostained with $\mathrm{TuJ} 1$, which recognizes neurons undergoing terminal mitosis and accumulates with further differentiation (Kim et al. 1997). Myt1is upregulated in gliomas (Armstrong et al. 1997) as well as following injury: spinal cord contusion (Wrathall et al. 1998), murine hepatitis virusinduced demyelination (Vana et al. 2007) and in multiple sclerosis lesions (Vana et al. 2007).

An important tool for establishing the in vivo role of transcription factors whose expression patterns implicate them in oligodendrocyte development is loss-of-function transgenic mice. Loss of Myt1 is hypothesized to affect not only the commitment to the oligodendrocyte lineage but also the subsequent differentiation of oligodendrocyte progenitors and the normal myelinating functions of mature oligodendrocytes. As suggested by in vitro overexpression studies, the $\mathrm{Myt1}^{-1-}$ may have reduced proliferative capacity as well as altered differentiation abilities (Nielsen et al. 2004). The Myt1 null mice may also be expected to suffer from neuronal dysfunction as suggested from the detection of Myt1 in neural precursors and selected populations of mature neurons, including motor neurons (Armstrong et al. 1995). In this paper we describe the construction and initial characterization of Mytl knock-in mice.

\section{Materials and methods}

\section{Construction of the Myt1 targeting vector}

The murine myelin transcription factor 1 (Myt1) gene is MGI:1100535 located on Chr2:181498037181562502 bp. Synonyms include: mKIAA0835, NZF-2a, NZF-2b, Nzf2, Nztf2. A building vector containing FLP recombinase target binding sites (frt) flanking a neomycin gene as well as a Cre recombinase target binding sites (loxP; pOSfrtLoxP) was supplied by Dr. Randy Thresher, University of North Carolina. The following cassettes were subcloned into this building vector:

1. the $4.9 \mathrm{~kb} \mathrm{Myt1} 5^{\prime}$ region of homology, which corresponds to the portion of intron 1 that ends at exon 2. This segment was cloned from $129 / \mathrm{SvEv}$ mouse genomic DNA using the forward primer 5'TATATATAGCGGCCGCTTACCAGGTAGC 
CTAATTGG3' and the reverse primer $5^{\prime}$ GTTA ATCGGCCGTTCTGAGCTCACCTGCAAAG3'.

2. the $e G F P$ gene subcloned from peGFP-C2 (Clontech; Mountain View, CA) using HindIII and KpnI.

3. the $i$ Cre recombinase subcloned from pBlue.iCre ((Shimshek et al. 2002); gift of R. Sprengel) using HindIII and KpnI. The iCre was first inserted upstream of the SV40 polyadenylation site in peGFP-C2 and in frame with GFP in order to create an eGFP-iCre fusion protein. The coding region of the fusion protein is directly followed by the polyadenylation site.

4. the $1.4 \mathrm{~kb} \mathrm{Mytl} 3^{\prime}$ region of homology, which corresponds to intron 2 and exon 3 . This segment was cloned from 129/SvEv mouse genomic DNA using the forward primer 5'GCATCGATTCAGT GCCAATGCAGTCTGGCC $3^{\prime}$ and the reverse

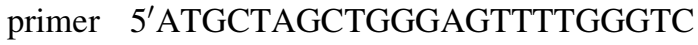
ATTGTGG3'.

To ensure initiation of translation with the eGFPiCre fusion protein, the Myt 1 initiation codon located at the beginning of exon 2 was changed to a GTG. The final $14.548 \mathrm{~kb}$ plasmid was sequenced in toto, which also confirmed that premature initiation of translation would not occur upstream of eGFP.

The NIH Institutional Biosafety Committee approved the Recombinant DNA Registration Document RD-95-I-06 for the use of this construct in mice.

Derivation and maintenance of transgenic mice

Electroporation of $129 / \mathrm{SvEv}$ embryonic stem cells with the Myt1 targeting construct was carried out by Dr. Randy Thresher at the University of North Carolina Animal Models Core Facility. Clones were screened by PCR using the forward primer: 5'CACACGC GTCACCTTAATATGCGAAG3' and the reverse primer: 5'CAGCAAGCCCTTTGTAAGTCACAGT $\mathrm{C}^{\prime}$ ' in order to amplify a $3.4 \mathrm{~Kb}$ band in transgenic animals.

We carried out Southern analysis to identify the correctly targeted ES cells, which were subsequently implanted at UNC. Four founders were then transferred to NIH and two lines (Myt1 A2A2 and Myt1 $\mathrm{C} 6 \mathrm{H} 5)$ were selected for detailed analysis. To remove the neomycin cassette, mice were bred for 5 generations with B6;SJL-Tg(ACTFLPe)9205Dym/J mice
(Jackson Laboratories, Bar Harbor, Maine). This transgenic strain expresses a variant of the Saccharomyces cerevisiae $F L P 1$ recombinase gene under the direction of the human ACTB promoter. The FLP1 recombinase excises the DNA sequence flanked by $f r t$ sites.

Southern and PCR analysis was carried out to assess whether recombination had occurred correctly at the Myt1 locus and to document the removal of the neomycin cassette. For the PCR analysis of resulting mice, the forward primer 5'ATACGAAGTTATTC GAAGTTCCTATTCTCTAGAAAG3' and reverse primer 5'ACCCTTAAATACTGCATCAAGGAACAA TCGAGGAGC $3^{\prime}$ detected a 1,435 bp band in mice containing the neomycin sequence and a $230 \mathrm{bp}$ band in mice lacking the neomycin sequence.

Neo- animals were back-crossed for at least 10 generations against C57BL/6 mice. Each generation was genotyped by PCR, and periodically confirmed with Southern Blot experiments. The final backcrosses are maintained as two heterozygous lines lacking neomycin (Myt1+/Myt1-, neo-/neo-). All the breedings are documented in a computerized Filemaker-based animal breeding database. Animals were handled according to IACUC guidelines under animal protocol \#1155-04. These "Myt1GFPicreKnockIn" mice are freely available through the Mutant Mouse Regional Resource Centers (MMRRC) under the MMRRC ID\#:031888.

Southern/Northern analysis and routine genotyping

Southern analysis was conducted on DNA purified from liver or whole embryos using a standard phenol extraction followed in some cases by a DNeasy treatment (Qiagen; Valencia, CA). Following restriction enzyme treatment, DNA was electrophoresed on $0.7 \%$ agarose gels. The marker lane consisted of the $1 \mathrm{~kb}$ DNA ladder Plus (Fermentas; Burlington, Ontario). Gels were pretreated with $0.2 \mathrm{~N} \mathrm{HCl}$ for 10 min, denatured in $1.5 \mathrm{~N} \mathrm{NaCl} / 0.5 \mathrm{~N} \mathrm{NaOH}$ for $45 \mathrm{~min}$, and neutralized in $1.5 \mathrm{~N} \mathrm{NaCl} / 1 \mathrm{M}$ Tris pH7.4 for 30 min prior to capillary transfer onto a positively charged nylon transfer membrane Hybond$\mathrm{N}^{+}$(Amersham; Piscataway, NJ). Probes were radiolabeled with $\alpha^{32} \mathrm{P}$ dCTP (Roche; Branchburg, NJ), and included the following: a 605 bp probe from the Myt1 5'region of homology; a 791 bp probe from the 
Myt1 $3^{\prime}$ region of homology; a 800 bp neomycin probe (from pPNT, LDN 33). The Myt1 $5^{\prime}$ and $3^{\prime}$ homology probes were cloned from $129 / \mathrm{SvEv}$ genomic DNA using PfuUltra high fidelity DNA polymerase (Stratagene; Garden Grove, CA) with the following primers: For the $605 \mathrm{bp} 5^{\prime}$ homology probe, the forward primer was $5^{\prime}$ GTTCCAAGA CAGCTATTACATGGTGGC ${ }^{\prime}$, and reverse primer was 5'GGAATTTGTATCTCTCCAGGGTCACTG3'. For the $791 \mathrm{bp} 3^{\prime}$ homology probe, the forward primer was $5^{\prime}$ GACTGTGACTTACAAAGGGCTT GCTG3', and the reverse primer was $5^{\prime} \mathrm{CTGACT}$ GATGGGTACCTCTCAATAGG3'. Each PCR fragment was subcloned into the pCR-Blunt II TOPO vector (Invitrogen; Carlsbad, CA). The 605 bp 5' homology probe can be excised from the pCR5'Myt1outSB605 plasmid with EcoRI, and the 791 bp $3^{\prime}$ homology probe can be excised from the pCR3'Myt1outSB791 plasmid with NotI and KpnI. Hybridizations were carried out in Hybrisol I solution (Millipore; Billerica, MA) with $100 \mu \mathrm{g} / \mathrm{ml}$ salmon sperm DNA (Phoenix Bio Tech; Phoenix, AZ).

\section{Northern analysis}

RNA was prepared from E15 brains by homogenization in TRIzol reagent (Invitrogen) using an IKA Ultra-Turrax T8 power homogenizer, followed by precipitation with isopropyl alcohol. RNA was electrophoresed on $1 \%$ agarose gels containing ethidium bromide together with a RNA Millenium molecular weight marker (Ambion; Austin, TX), and blotted with NorthernMax-Gly reagents (Ambion). Capillary transfered onto positively charged nylon transfer membrane Hybond- $\mathrm{N}^{+}$(Amersham). Probes were radiolabeled with $\mathrm{a}^{32} \mathrm{P}$ dCTP (Roche; Branchburg, NJ) and included the following: a $580 \mathrm{bp}$ cDNA probe, cloned from pmMyt1 plasmid containing murine Myt1 cDNA using PfuUltra high fidelity DNA polymerase (Stratagene) with the forward primer: $5^{\prime}$ GAATTCGGAGCTCTCAATGGTTCATC $3^{\prime}$, and the reverse primer: $5^{\prime}$ GAATTCCAG CTTGCTTGATCGTTTCC $3^{\prime}$. This $580 \mathrm{bp}$ PCR fragment was then subcloned into the pCR-Blunt II TOPO vector (Invitrogen) to generate the pCRMyt1NB580 plasmid. The 580 bp Myt1 cDNA fragment can be excised from the Myt1NB580 plasmid with EcoRI or directly labeled with $\alpha^{32} \mathrm{P}$ dCTP (Roche). Hybridizations were carried out in Hybrisol I solution (Millipore) with $100 \mu \mathrm{g} / \mathrm{ml}$ salmon sperm DNA (Phoenix Bio Tech).

\section{Genotyping by PCR}

Tail or toe lysates were prepared by incubating samples from 3-16 h in $50 \mu \mathrm{l}$ of $1 \mathrm{M}$ Tris $\mathrm{pH} 8.0$, $5 \mathrm{M} \mathrm{NaCl}, 0.5 \mathrm{M}$ EDTA, $10 \%$ Tween 20, 10\% NP$40,0.4 \mu \mathrm{g} / \mathrm{ml}$ freshly added proteinase K. Following centrifugation to remove debris, $1 \mu \mathrm{l}$ of DNA lysate was added to $0.1 \mu \mathrm{l}$ of each primer ( $0.4 \mu \mathrm{l}$ total), 0.2 $\mu \mathrm{l}$ Taq DNA polymerase (Promega), $5 \mu 1 \%$ Triton $\mathrm{X}-100$ and $18.4 \mu \mathrm{l} \mathrm{H}_{2} \mathrm{O}$ in EasyStart Micro 50 tubes (M $\beta$ P; San Diego, CA). Two primer pairs were used in each tube in order to discriminate between transgenic homozygous $\left(M y t \mathrm{I}^{-/-}\right)$, heterozygous $\left(M y t 1^{ \pm}\right)$, and wild-type mice $\left(M y t 1^{+/+}\right)$. To amplify a $325 \mathrm{bp}$ transgenic fragment, the forward primer was $5^{\prime}$ CAGCCATACCACATTTGAGAGG3' and the reverse primer was $5^{\prime}$ TGCATTGGCACTGAATC GATGG3'. To amplify a 188 bp wild type fragment, the forward primer was $5^{\prime C C A A C T T G C T T C T T T ~}$ TGGTCCC $3^{\prime}$ and the reverse primer was $5^{\prime}$ GGCT CGCTTGTCATCACTTTC $3^{\prime}$. The PCR cycling was carried out for a total of 30 cycles in a Hybaid, PCR Express HBPX110 (Thermo; Essex, UK) under the following conditions: melt at $94^{\circ} \mathrm{C}$ for $5 \mathrm{~min}$, denature at $94^{\circ} \mathrm{C}$ for $30 \mathrm{~s}$, anneal at $55^{\circ} \mathrm{C}$ for $30 \mathrm{~s}$, extend $72^{\circ} \mathrm{C}$ for $1 \mathrm{~min}$ (30 cycles). PCR products were analyzed on a $2 \%$ agarose gel containing ethidium bromide. The marker lane consists of the $100 \mathrm{bp}$ DNA ladder Plus (Fermentas). In this assay, $\mathrm{Mytl}^{-1-}$ mice display a single $325 \mathrm{bp}$ band, Myt $1^{ \pm}$display both the $325 \mathrm{bp}$ and $188 \mathrm{bp}$ bands, and $M y t 1^{+/+}$mice display a single 188 bp band.

Immunohistochemistry and statistical analysis

E18 spinal cords were immersion fixed in phosphatebuffered $4 \%$ paraformaldehyde with picric acid (FD Neurotechnologies; Baltimore, MD). Following cryoprotection treatment in $0.1 \mathrm{M}$ phosphate buffer $(\mathrm{pH}$ 7.4) containing $20 \%$ sucrose for $72 \mathrm{~h}$ at $4^{\circ} \mathrm{C}$, spinal cords were rapidly frozen and stored at $-75^{\circ} \mathrm{C}$. Serial cryostat sections $(20 \mu \mathrm{m})$ were cut transversely 
through the whole spinal cord. Every 1st, 2nd and 3rd section of each series of 10 sections were mounted on Superfrost Plus microscope slides (3 sections per slide). Upon drying, sections were stored at $-20^{\circ} \mathrm{C}$. Following washes in $0.01 \mathrm{M}$ phosphate-buffered saline (PBS; pH 7.4), sections were incubated overnight at $4^{\circ} \mathrm{C}$ in PBS containing $1 \%$ normal donkey serum, 4\% BSA (Jackson ImmunoResearch, West Grove, PA), 0.3\% Triton X-100 (Sigma, St. Louis, MO), the polyclonal rabbit anti-eGFP IgG (1:200, Invitrogen, Carlsbad, CA) and the biotinylated monoclonal mouse anti-NeuN antibody $(1: 100$, Millipore, Billerica, MA). This was followed by incubation of sections at room temperature in the PBS/Triton X solution containing Alexa Fluor $^{\circledR} 488$ donkey anti-rabbit IgG (Molecular Probe, Eugene, OR) for $1 \mathrm{~h}$, and then in the PBS/Triton X containing streptavidin conjugated Alexa Fluor ${ }^{\circledR} 594$ (Molecular Probe) for another hour. Each step was followed by washes in PBS. Sections were coverslipped using Vectashield ${ }^{\circledR}$ with DAPI (Vector Labs, Burlingame, CA) and stored at $4^{\circ} \mathrm{C}$.

Photographs of fixed 18-day mouse embryo spinal cord sections were captured with the Olympus $1 \times 70$ fluorescent microscope using the $4 \mathrm{X}$ UPLanF1 objective lens. The photographs were processed and digitized using the CoolSNAP fX camera (Photometrics, Roper Scientifics, Tucson, $\mathrm{AZ}$ ) and the IP Lab version 3.9.5 rl program (Scientific Image Processing, BDBiosciences, San Jose, CA). The photographs were further processed with Adobe Photoshop CS3, Version 10.0.1 (Adobe Systems Inc., San Jose, CA) utilizing the Channel window to prepare separate fluorescent images for the eGFP (Alexa Fluor ${ }^{\circledR} 488$ ), NeuN (Alexa Fluor ${ }^{\circledR} 594$ ) and DAP.

Cells that fluoresced for eGFP, NeuN and DAPI were counted using the ImageJ program (Rasband, W.S., ImageJ, National Institutes of Health, Bethesda, MD, USA, http://rsb.info.nih.gov/ij/ 1997-2009 (also see Abramoff et al. 2004). Two separate sets of 18 day embryo spinal cords from the wild type, heterozygote and homozygote animals were counted. The cells in fourteen sections representing 14 different areas of each 18 day mouse embryo spinal cord (wild type, heterozygote and homozygote) from the cervical to sacral were counted. The 2 sets were averaged and graphed with Microsoft Excel (Microsoft Corp., Redmond, WA).

\section{Results}

Generating Mytl knock-in mice

Myt1 can be transcribed from two alternative promoters to yield either a 6 zinc finger protein (6ZFMyt1 or Myt1a) or a 7 zinc finger protein (7ZFMyt1 or Myt1b; Matsushita et al. 2002; Nielsen et al. 2004). 7ZFMyt1 is by far the predominant product of the Myt1 locus in all tissues examined (Matsushita et al. 2002), (Nielsen et al. 2004); (Gu et al. 2004). To establish the in vivo role of Myt1, we designed a knock-in construct so that the promoter for the seven zinc finger Myt1 isoform (7ZFMyt1) would drive expression of a green fluorescent protein (eGFP) reporter together with the cre recombinase (Fig. 1). Cassettes for these two reporter genes were fused in frame, so that translation of the eGFP-iCre fusion protein would occur from the eGFP-iCre initiation codon within exon 2. The initiation codon for 7ZFMyt 1 in exon 2 was altered. Also included was a neo selectable marker flanked with frt sites so that the neo cassette, which can impact the expression of the targeted gene, could be subsequently excised by crossing transgenic mice with mice expressing the flip recombinase.

Correctly recombined transgenes were documented in two lines of Myt1 knock-in mice by Southern analysis (Fig. 2). Using a probe that is distal to the $5^{\prime}$ region of homology (panel b), the expected $5.9 \mathrm{~kb} K p n \mathrm{I}$ band (lanes 2 and 3) and $6.3 \mathrm{~kb}$ HindIII band (lanes 5 and 6) were observed in both $\mathrm{Myt}^{ \pm}$, $\mathrm{Neo}^{ \pm}$and $\mathrm{Myt}^{-/-}, \mathrm{Neo}^{-/-}$mice. Using a probe that is distal to the $3^{\prime}$ region of homology (panel a), the expected $8.4 \mathrm{~kb}$ HindIII band was observed in $\mathrm{Myt}^{ \pm}, \mathrm{Neo}^{ \pm}$(lane 2) and $\mathrm{Myt}^{-/-}, \mathrm{Neo}^{+/+}$(lane 3) animals. This band was also detectable with a neo probe (lane 8). A smaller HindIII band of $7.2 \mathrm{~kb}$ was observed in animals that were derived from matings with mice expressing the flip recombinase (lanes 4 and 5), indicating that neo had been excised from the targeted Myt1 locus in these animals. The $5^{\prime}$ region of homology probe and the 3 'region of homology probe together confirm the correct homologous recombination of the electroporated knock-in construct.

No message corresponding to Myt1 was detectable by Northern analysis in homozygous Myt1 knock-in mice at embryonic day 15 , a time when Myt1 is normally highly expressed (lanes 3 and 6, Fig. 3). 

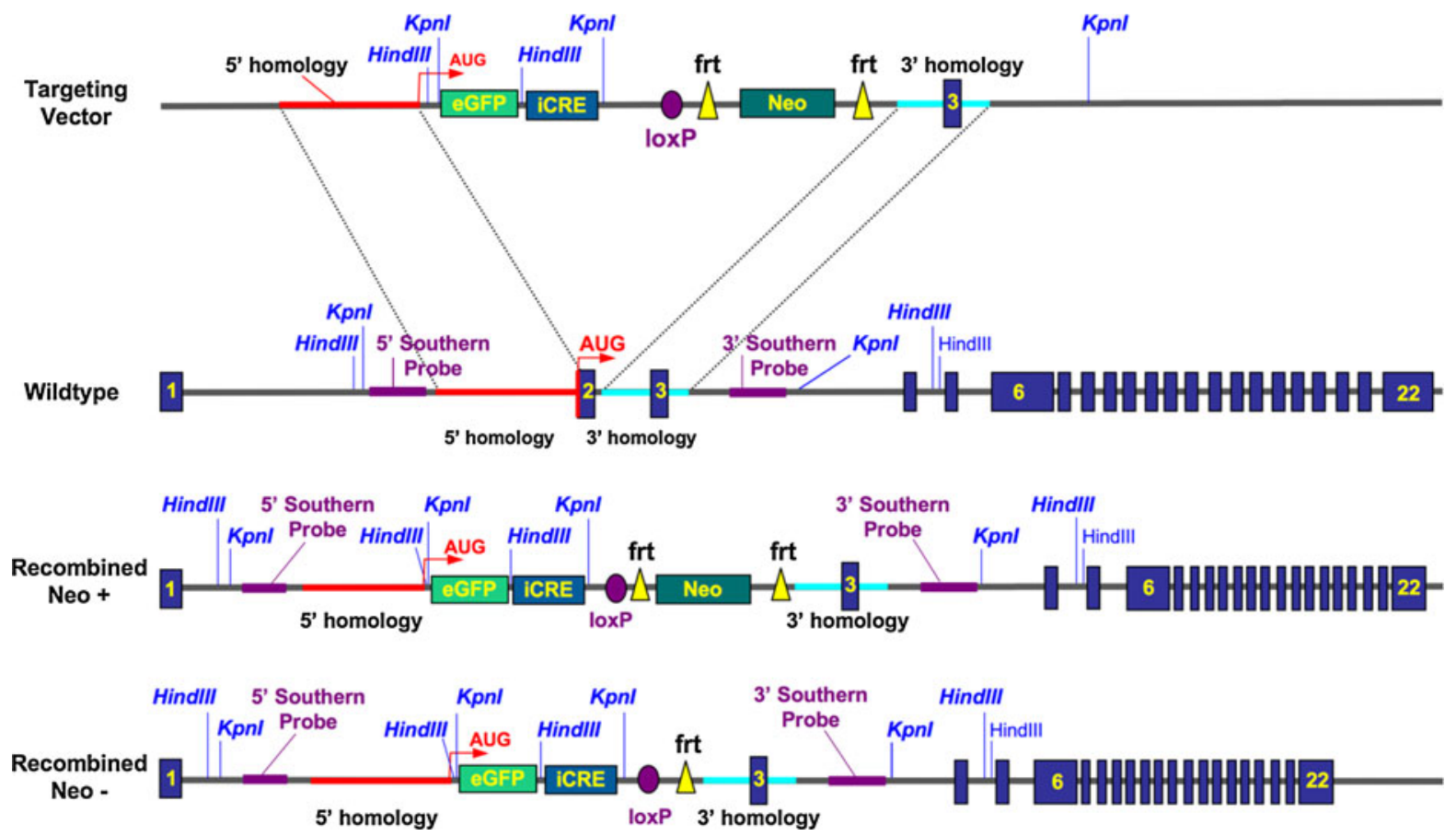

Fig. 1 Construction of Myt1 knock-in Mice. The targeting vector is designed to eliminate exon 2 , which contains the initiation codon for 7ZFMyt1, (depicted as AUG with a red arrow) and drive expression of the eGFP/cre fusion gene directly from the 7ZFMyt 1 promoter. The probes used on Southern blots to document the recombinant event are shown in pink and are distal to the $5^{\prime}$ region of homology (in red) and the $3^{\prime}$ region of homology (in light blue). The frt recombination sites flanking the Neomycin (Neo) are marked with triangles. Note that following excision of the neomycin cassette by breeding with mice expressing the flip recombinase, a single frt site remains, together with a loxP site. The 22 exons are pictured as boxes (not drawn to scale). (Color figure online)
A Myt1 $+/++/-\%+\%+\% \quad+/+\%$ $\begin{array}{llllllllll}M & 1 & 2 & 3 & 4 & 5 & 6 & 7 & 8 & 9\end{array}$

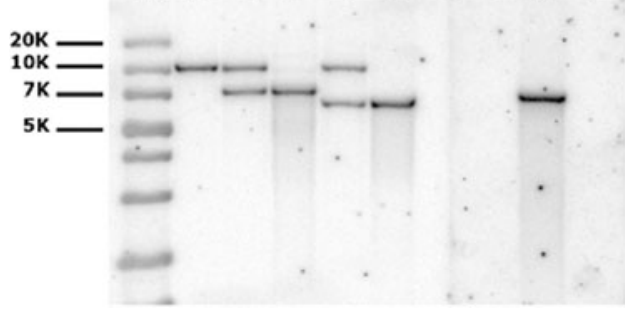

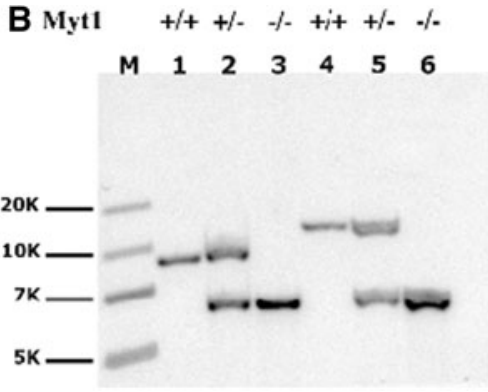

identifies a $7.2 \mathrm{~kb}$ HindIII band. In panel b, KpnI (lanes 1-3) or HindIII-digested (lanes 4-6) genomic DNA was hybridized with the $5^{\prime}$ Myt 1 outside region of homology probe. lane 1 $\mathrm{Myt}^{+/+}, \mathrm{Neo}^{-/-}$; lane $2 \mathrm{Myt}^{ \pm}, \mathrm{Neo}^{ \pm}$; lane $3 \mathrm{Myt}^{-/-}$, $\mathrm{NeO}^{+/+}$; lane $4 \mathrm{Myt}^{+/+}$, $\mathrm{Neo}^{-/-}$; lane $5 \mathrm{Myt}^{ \pm}$, $\mathrm{Neo}^{ \pm}$; lane 6 $\mathrm{Myt}^{-/-}, \mathrm{Neo}^{+/+}$. The $5^{\prime} \mathrm{Myt} 1$ probe identifies a $8.7 \mathrm{~kb}$ KpnI band in wild type mice and a $5.9 \mathrm{~kb} \mathrm{KpnI}$ band in transgenics. It also reveals a $12.2 \mathrm{~kb}$ HindIII band in wild type mice and a $6.3 \mathrm{~kb}$ HindIII band in transgenics. Both panels include a $1 \mathrm{~kb}$ DNA ladder Plus as a molecular weight marker 
Heterozygous embryos expressed approximately half as much of the $5.1 \mathrm{~kb}$ Myt1 transcripts at E15 when either total RNA (lane 2) or polyA-selected RNA (lane 5) was analyzed.

\section{Phenotype of Mytl knock-in mice}

In our initial PCR genotyping and Southern blot analysis of transgenic animals, no homozygous animals were present in litters analyzed at postnatal day 7. To determine if there was a viability problem with homozygous animals, and to pinpoint the time at which survival may be affected, we set up a series of matings between heterozygous animals. After collecting embryos throughout prenatal development, we determined that homozygous animals survived through the prenatal period but died shortly after birth. Table 1 summarizes the results from an embryonic and a postnatal time point. At embryonic day 18 , the observed numbers of wild type, homozygous and heterozygous closely approximate the expected Mendelian ratio, with $26.8 \%$ of the offspring representing homozygous animals. No living homozygous animals were found from the 15 matings analyzed at birth or the 18 matings that were analyzed at later postnatal ages. One mutant allele had no affect on viability.

To determine the cause of death, heterozygous animals were mated and a Caesarean section performed shortly before the scheduled birth. Pups were kept at $37^{\circ} \mathrm{C}$ during the hour observation period. Unlike wild type or heterozygous animals, the homozygous animals all died within $20 \mathrm{~min}$. Homozygous animals never progressed from gasping to normal breathing. Their lungs did not appear white in color and were not inflated as evidenced by their lack of buoyancy when placed in a container of phosphate buffered saline.

Heterozygous animals appeared undistinguishable from wild type animals and reproduced normally.

Neural cells with an active Myt1 promoter can be tracked with eGFP in knock-in mice

Myt1 expressing cells in null or heterozygous embryos can be identified through the eGFP marker (Fig. 4). At E18, eGFP cells are distributed throughout the spinal cord (panels c). These eGFP cells were
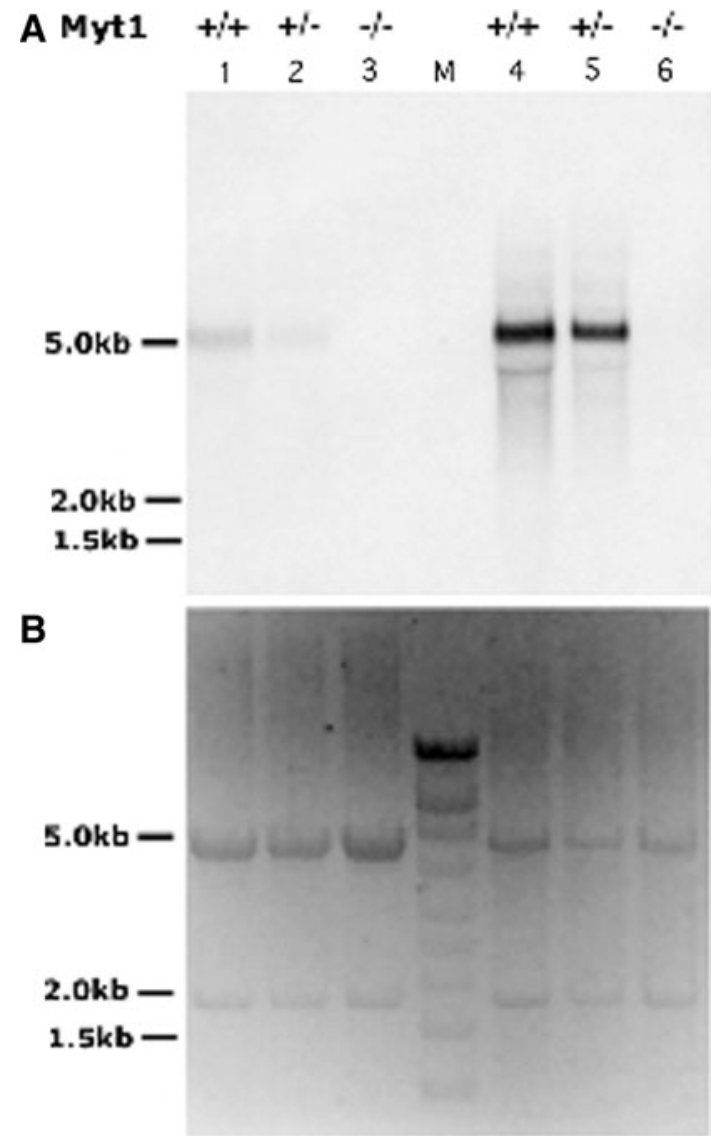

Fig. 3 Northern Analysis of the Myt1 knock-in Mice. This analysis was carried out after the crossing with B6;SJL$\mathrm{Tg}$ (ACTFLPe)9205Dym/J mice that express the Saccharomyces cerevisiae FLP1 recombinase gene. All mice were genotyped to verify that the neo gene was absent. In panel a, a Myt1 cDNA probe identified a $5.1 \mathrm{~kb}$ band in either total RNA (lanes 1-3) or mRNA (lanes 4-6) in mice containing at least one wild-type Myt1 allele. lane $1 \mathrm{Myt}^{+/+}$, lane $2 \mathrm{Myt}^{ \pm}$, lane $3 \mathrm{Myt}^{-1-}$, RNA Millenium molecular weight marker; lane $4 \mathrm{Myt}^{+/+}$, lane $5 \mathrm{Myt}^{ \pm}$, lane $6 \mathrm{Myt}^{-/-}$. No Myt1 transcripts in any size range are detectable in the null mice. In panel $\mathbf{b}$, the ethidium bromide stained gel shows the integrity of the RNA samples

not double labeled with Neuronal Nuclei protein (NeuN; panels a), suggesting that Myt1 neural progenitors have not differentiated into the mature neurons identified by NeuN staining at this age. Moreover, the pattern of NeuN staining in transgenic embryos was similar to that in wild type embryos (panels b). The lack of overlap between eGFPpositive cells and NeuN cells is shown in a higher 
Table 1 Genotyping the progeny from Myt $1 \pm$ Matings

\begin{tabular}{|c|c|c|c|c|c|c|}
\hline \multirow[b]{2}{*}{ Genotype } & \multicolumn{3}{|c|}{ E18 embryos (from 48 matings) } & \multicolumn{3}{|c|}{ Postnatal day 7 mice (from 18 matings) } \\
\hline & $M y t 1+/+$ & $M y t 1 \pm$ & Myt1-/- & $M y t 1+/+$ & $M y t 1 \pm$ & Myt1-/- \\
\hline Number of offspring & 102 & 190 & 107 & 37 & 52 & 0 \\
\hline$\%$ of offspring & 25.6 & 47.6 & 26.8 & 41.6 & 58.4 & 0 \\
\hline
\end{tabular}
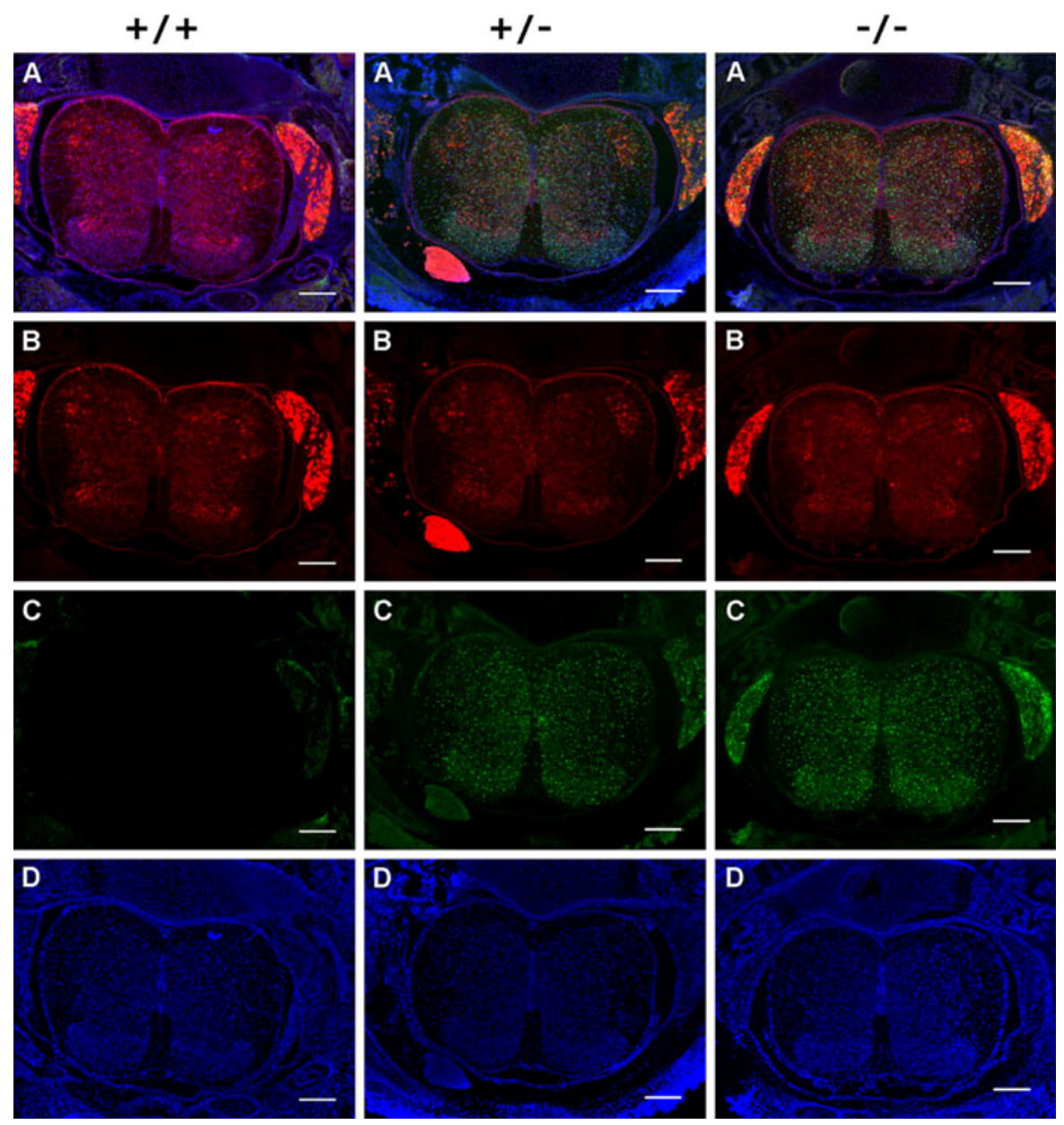

Fig. 4 Immunohistochemistry of Spinal Cord Sections from Myt1 knock-in Mice. Spinal cord sections from E18 wild type mice $(+/+)$, heterozygotes $( \pm)$ or null mice $(-/-)$ were triple stained with: panel a, eGFP (green), NeuN (red) and DAPI.

magnification view of a heterozygous spinal cord (Fig. 5). Quantitation of the immunohistochemistry is shown in Fig. 6. No significant difference in the number of NeuN cells was apparent in null or heterozygous animals compared to wild type. Likewise, there was no significant different in the number of eGFP cells in heterozygous or null animals. panel b shows NeuN alone; panel c shows eGFP alone; and panel $\mathbf{d}$ shows DAPI alone. The size bar $=10$ microns. (Color figure online)

\section{Discussion}

The Myt1 knock-in mice should provide an invaluable resource for: (1) defining the role of the Myt1 gene family in the specification of neural lineages and the development of oligodendrocytes, and (2) determining the set of gene targets that this structurally 


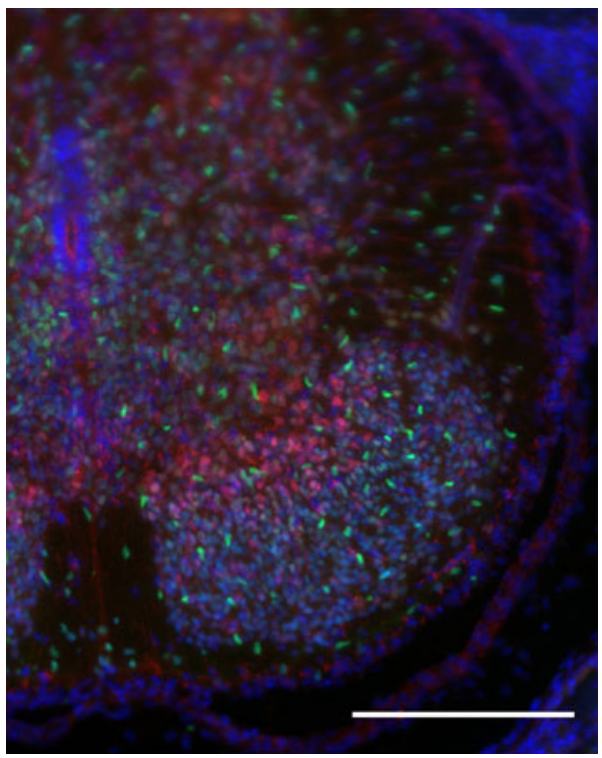

Fig. 5 Immunohistochemistry of Spinal Cord Sections from Myt1 knock-in Mice. A spinal cord section from anE18 homozygote $(-/-)$ mouse was triple stained with eGFP (green), NeuN (red) and DAPI. The size bar $=10$ microns. (Color figure online)

unique class of zinc finger transcription factors binds to in vivo. Recently, attention to the Myt1 gene family has focused on the discovery that the Myt1 paralog, Myt11, is one of a simple trio of genes necessary for the efficient conversion of mouse fibroblasts into functional neurons in vitro (Vierbuchen et al. 2010). While documenting that a Myt1 gene family member is instrumental in neural specification, this advance is short on the molecular details of what genes are activated or repressed and in what stages of the cells. In the Myt1 knock-in mice, lineage tracing is possible due to the incorporation of a cre coding region fused in frame to the GFP reporter. Heterozygous transgenic animals mated to the ROSA26 Cre indicator strain (Soriano 1999) will display $\beta$-galactosidase expression in all Myt1 lineage cells. Additional Cre-reporter strains that would be helpful include the Z/AP line that contains a human placental alkaline phosphatase (hPLAP) reporter (Lobe et al. 1999) and the R26R-EYFP line with an enhanced yellow fluorescent protein (EYFP) reporter (Srinivas et al. 2001).

Elimination of Myt1 expression disrupts neural development. Another Myt1 transgenic model constructed by $\mathrm{Gu}$ and colleagues displayed a breathing defect attributable to improper innervation of the

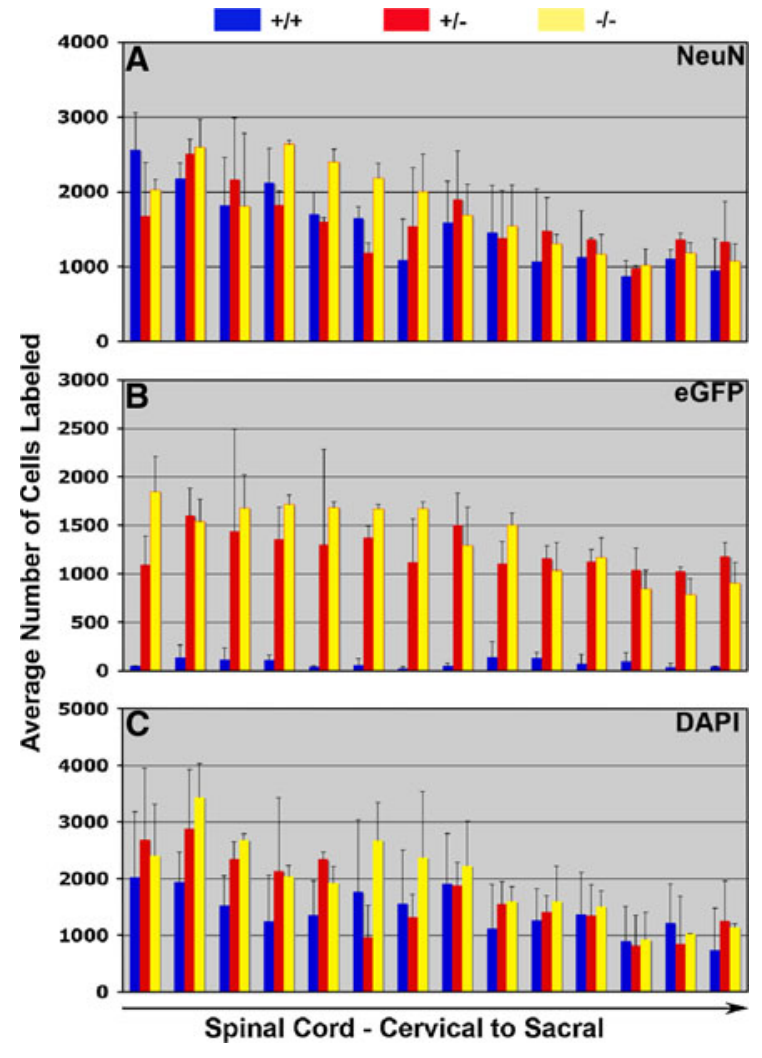

Fig. 6 Quantitation of Immunohistochemistry. The average of the total number of cells labeled with NeuN, eGFP or DAPI per E18 spinal cord section are shown for wild type mice $(+/+$; blue bars), heterozygotes ( \pm ; red bars) or null mice (-I-; yellow bars). Black lines represent standard deviations. (Color figure online)

diaphragm in the transgenic mice (Wang et al. 2007). Likewise, the homozygous knock-in Myt1 mice in the present study presented with breathing difficulties shortly after birth. Given that the transgenic line constructed by $\mathrm{Gu}$ and colleagues is potentially a dominant negative model, since altered Myt1 transcripts are present (Wang et al. 2007), it is reassuring that this phenotype is seen in both transgenic models. The new data provided in the present study includes the observation that eliminating Myt1 does not affect the number or distribution of neurons. These data would not have been predicted based on earlier observations that Myt1 is expressed by rat cortical neuronal progenitors at E11 and is up-regulated 2.7fold between E11 and E13 (Nielsen et al. 2009). In the Myt1 eGFP/cre knock-in mice, the distribution and number of differentiated neurons in the spinal cord appears normal, as does the pattern of neural 
cells in which the Myt1 promoter actively drives the expression of the eGFP reporter (Figs. 4, 5 and 6). Of note, the eGFP reporter is not expressed by the neuronal population labeled by NeuN in the spinal cord. Oligodendrocytes and motor neurons were thought to derive from a common precursor expressing Olig 1 and Olig 2 (Zhou and Anderson 2002; Lu et al. 2000), but subsequent fate mapping studies identified multiple sources generating oligodendrocytes in the brain and spinal cord (Kessaris et al. 2006; Cai et al. 2005). Further lineage tracing studies with the cre reporter may help resolve the origin of some of these oligodendrocyte subpopulations.

Myt1 is also critical for islet cell differentiation, as shown in a series of elegant experiments by $\mathrm{Gu}$ and Hecksher-Sorensen and their colleagues (AhnfeltRonne et al. 2007; Wang et al. 2007, 2008). The lossof-function Myt1 model constructed by targeting exon 9 has a Myt1 mRNA level reduced by over fourfold; heterozygous mice displayed normal pancreatic development, making it unlikely that the Myt1- allele behaves in a dominant negative fashion in this transgenic model (Wang et al. 2007). Compensatory activation of the paralogs Myt11 and Myt3 occurs in the embryonic $\mathrm{Myt1}^{-/-}$pancreas. Of particular interest is the observation that Myt1 and neurogenin 3 (Ngn3) positively regulate each other's expression in the developing pancreas, with Notch mediated signaling regulating the number of Ngn3+ cells. A Notch signaling pathway is likewise a feature of the switch from neurogenesis to gliogenesis in the spinal cord, which involves not only the induction of Olig2 expression, but also the down-regulation of neurogenin $1(\mathrm{Ngn} 1)$ and neurogenin $2(\mathrm{Ngn} 2)$ via Notch signaling (Zhou et al. 2001).

Genomic approaches to oligodendrocyte biology have greatly expanded our horizon by showing the multiple different temporal transcription factor expression patterns occurring during oligodendrocyte differentiation. (Dugas et al. 2006; Nielsen et al. 2006). While Myt1 is down-regulated during oligodendrocyte differentiation, Myt is in the company of sixty other transcription factors that are up-regulated over twofold in cortical neuronal progenitors between embryonic day 11 and 13 (Nielsen et al. 2009). Seventy percent of the transcription factors up-regulated in cultured oligodendrocytes during differentiaton in the absence of neurons are also up-regulated in acutely isolated developing oligodendrocytes (Dugas et al. 2006; Nielsen et al. 2006). Many of these transcription factors await functional studies before their node in the complex network regulating oligodendrocyte development can be assigned. In Myt1 knock-in mice, the absence of Myt1 is expected to lead to the inactivation of an array of target genes. Transgenic mice provide a handle for identifying target genes of transcription factors when combined with the powerful "omics" technologies to identify which transcripts are over or under-expressed in the presence or absence of a given transcription factor. Given the wealth of molecular detail available on how Myt1 burrows into the DNA groove and recruits histone deacetylase to regulate neural transcription, the Myt1 knock-in mice offer an invaluable tool for further exploration of the transcriptional circuitry in neural cells.

Acknowledgments The authors thank Dr. Eva Mezey for insights on quantitative immunohistochemistry, Wayne Rasband for assistance with the imageJ program, and Dr. Heinz Arnheiter for training and access to his computerized Filemaker-based animal breeding database program. Dr. Gabor Lovas' insights on the phenotype of the knock-in mice are gratefully acknowledged. This work was supported by National Institute of Neurological Disorders and Stroke (NINDS)/ National Institutes of Health intramural funds to LDH for project\# 1 Z01 NS002528.

Open Access This article is distributed under the terms of the Creative Commons Attribution Noncommercial License which permits any noncommercial use, distribution, and reproduction in any medium, provided the original author(s) and source are credited.

\section{References}

Abramoff MD, Magelhaes PJ, Ram SJ (2004) Image processing with image. J Biophotonics Int 11(7):36-42

Ahnfelt-Ronne J, Hald J, Bodker A, Yassin H, Serup P, Hecksher-Sorensen J (2007) Preservation of proliferating pancreatic progenitor cells by Delta-Notch signaling in the embryonic chicken pancreas. BMC Dev Biol 7:63

Armstrong RC, Kim JG, Hudson LD (1995) Expression of myelin transcription factor I (MyTI), a "zinc-finger" DNA-binding protein, in developing oligodendrocytes. Glia 14:303-321

Armstrong RC, Migneault A, Shegog ML, Kim JG, Hudson LD, Hessler RB (1997) High-grade human brain tumors exhibit increased expression of myelin transcription factor 1 (MYT1), a zinc finger DNA-binding protein. J Neuropathol Exp Neurol 56:772-781

Bellefroid EJ, Bourguignon C, Hollemann T, Ma Q, Anderson DJ, Kintner C, Pieler T (1996) X-MyT1, a Xenopus 
C2HC-type zinc finger protein with a regulatory function in neuronal differentiation. Cell 87:1191-1202

Cai J, Qi Y, Hu X, Tan M, Liu Z, Zhang J, Li Q, Sander M, Qiu M (2005) Generation of oligodendrocyte precursor cells from mouse dorsal spinal cord independent of Nkx6 regulation and Shh signaling. Neuron 45:41-53

Dugas JC, Tai YC, Speed TP, Ngai J, Barres BA (2006) Functional genomic analysis of oligodendrocyte differentiation. J Neurosci 26:10967-10983

Gamsjaeger R, Swanton MK, Kobus FJ, Lehtomaki E, Lowry JA, Kwan AH, Matthews JM, Mackay JP (2008) Structural and biophysical analysis of the DNA binding properties of myelin transcription factor 1. J Biol Chem 283:5158-5167

Gu G, Wells JM, Dombkowski D, Preffer F, Aronow B, Melton DA (2004) Global expression analysis of gene regulatory pathways during endocrine pancreatic development. Development 131:165-179

Kessaris N, Fogarty M, Iannarelli P, Grist M, Wegner M, Richardson WD (2006) Competing waves of oligodendrocytes in the forebrain and postnatal elimination of an embryonic lineage. Nat Neurosci 9:173-179

Kim JG, Hudson LD (1992) Novel member of the zinc finger superfamily: a $\mathrm{C} 2-\mathrm{HC}$ finger that recognizes a glia-specific gene. Mol Cell Biol 12:5632-5639

Kim JG, Armstrong RC, v Agoston D, Robinsky A, Wiese C, Nagle J, Hudson LD (1997) Myelin transcription factor 1 (Myt1) of the oligodendrocyte lineage, along with a closely related CCHC zinc finger, is expressed in developing neurons in the mammalian central nervous system. J Neurosci Res 50:272-290

Lobe CG, Koop KE, Kreppner W, Lomeli H, Gertsenstein M, Nagy A (1999) Z/AP, a double reporter for cre-mediated recombination. Dev Biol 208:281-292

Lu QR, Yuk D, Alberta JA, Zhu Z, Pawlitzky I, Chan J, McMahon AP, Stiles CD, Rowitch DH (2000) Sonic hedgehog-regulated oligodendrocyte lineage genes encoding bHLH proteins in the mammalian central nervous system. Neuron 25:317-329

Matsushita F, Kameyama T, Marunouchi T (2002) NZF-2b is a novel predominant form of mouse NZF-2/MyT1, expressed in differentiated neurons especially at higher levels in newly generated ones. Mech Dev 118:209-213

Nicolay DJ, Doucette JR, Nazarali AJ (2007) Transcriptional control of oligodendrogenesis. Glia 55:1287-1299

Nielsen JA, Hudson LD, Armstrong RC (2002) Nuclear organization in differentiating oligodendrocytes. J Cell Sci 115:4071-4079

Nielsen JA, Berndt JA, Hudson LD, Armstrong RC (2004) Myelin transcription factor 1 (Myt1) modulates the proliferation and differentiation of oligodendrocyte lineage cells. Mol Cell Neurosci 25:111-123
Nielsen JA, Maric D, Lau P, Barker JL, Hudson LD (2006) Identification of a novel oligodendrocyte cell adhesion protein using gene expression profiling. J Neurosci 26:9881-9891

Nielsen JA, Lau P, Maric D, Barker JL, Hudson LD (2009) Integrating microRNA and mRNA expression profiles of neuronal progenitors to identify regulatory networks underlying the onset of cortical neurogenesis. BMC Neurosci 10:98

Romm E, Nielsen JA, Kim JG, Hudson LD (2005) Myt1 family recruits histone deacetylase to regulate neural transcription. J Neurochem 93:1444-1453

Shimshek DR, Kim J, Hubner MR, Spergel DJ, Buchholz F, Casanova E, Stewart AF, Seeburg PH, Sprengel R (2002) Codon-improved Cre recombinase (iCre) expression in the mouse. Genesis 32:19-26

Soriano P (1999) Generalized lacZ expression with the ROSA26 Cre reporter strain. Nat Genet 21:70-71

Srinivas S, Watanabe T, Lin CS, William CM, Tanabe Y, Jessell TM, Costantini F (2001) Cre reporter strains produced by targeted insertion of EYFP and ECFP into the ROSA26 locus. BMC Dev Biol 1:4

Vana AC, Lucchinetti CF, Le TQ, Armstrong RC (2007) Myelin transcription factor 1 (Myt1) expression in demyelinated lesions of rodent and human CNS. Glia 55:687-697

Vierbuchen T, Ostermeier A, Pang ZP, Kokubu Y, Sudhof TC, Wernig M (2010) Direct conversion of fibroblasts to functional neurons by defined factors. Nature 463: 1035-1041

Wang S, Zhang J, Zhao A, Hipkens S, Magnuson MA, Gu G (2007) Loss of Myt1 function partially compromises endocrine islet cell differentiation and pancreatic physiological function in the mouse. Mech Dev 124:898-910

Wang S, Hecksher-Sorensen J, Xu Y, Zhao A, Dor Y, Rosenberg L, Serup P, Gu G, Ahnfelt-Ronne J, Hald J, Bodker A, Yassin H (2008) Myt1 and Ngn3 form a feedforward expression loop to promote endocrine islet cell differentiation Preservation of proliferating pancreatic progenitor cells by Delta-Notch signaling in the embryonic chicken pancreas. Dev Biol 317:531-540

Wegner M (2008) A matter of identity: transcriptional control in oligodendrocytes. J Mol Neurosci 35:3-12

Wrathall JR, Li W, Hudson LD (1998) Myelin gene expression after experimental contusive spinal cord injury. J Neurosci 18:8780-8793

Zhou Q, Anderson DJ (2002) The bHLH transcription factors OLIG2 and OLIG1 couple neuronal and glial subtype specification. Cell 109:61-73

Zhou Q, Choi G, Anderson DJ (2001) The bHLH transcription factor Olig2 promotes oligodendrocyte differentiation in collaboration with $\mathrm{Nkx} 2.2$. Neuron 31:791-807 